\title{
Short-arc Orbit Determination Method for GEO Satellite of Partial Subsatellite Point
}

\author{
Xiao-Jie $\mathrm{LI}^{1, \mathrm{a}^{*}}$, Rui GUO ${ }^{1, \mathrm{~b}}$, Jin HUANG${ }^{2, \mathrm{c}}$, Guang-Ming HU ${ }^{1, \mathrm{~d}}$, Ling-Feng \\ $\mathrm{ZHU}^{1, \mathrm{e}}$, Kui GENG ${ }^{3, \mathrm{f}}$ \\ ${ }^{1}$ China Beijing Satellite Navigation Center, Beijing 100094, China \\ ${ }^{2}$ Beijing Remote Sensing Institute, Beijing 100192, China \\ ${ }^{3}$ China Agriculture University, Yantai 264670, China \\ alxjant1984@126.com, 'buorui@shao.ac.cn, 'gold1983@qq.com, dhuguangming_nv@163.com, \\ ezlfchxy@tom.com, gengkui1992@163.com
}

${ }^{*}$ Corresponding author

\begin{abstract}
Keywords: Beidou System(BDS), Partial Subsatellite Point, Geostationary Satellite(GEO), Orbit Rapid Recovery.
\end{abstract}

\begin{abstract}
Subsatellite point for geostationary satellite(GEO) need to be retained based on the continual orbit maneuver, especially for GEO satellite of partial subsatellite point. How to recover orbit rapidly for GEO based on short-arc tracking is the key factor to improve the service performance and usability of the navigation system. Due to its geostationary feather, GEO orbit parameters are strongly correlated with the clock corrections parameters in precise orbit determination (POD) using L-band pseudorange data, which needs supports by the time synchronization technique. This paper bringed forward a orbit rapid recovery method based on satellite clock offset and station clock offset. The satellite-ground time synchronization were realized by satellite-earth wireless two-way method, ground-ground time synchronization were realized by constellation combining orbit determination method. The precise orbit determination was realized using pseudorange and phase data without clock offset. A new method of parameters decorrelation based on prior restriction and a short-arc orbit determination method based on restriction by long-arc orbit determination were brought forward. The experiment based on ground data of Beidou system(BDS) indicated that the time of orbit recovery after maneuver was shortened from $24 \mathrm{~h}$ to $4 \mathrm{~h}$. The 2-hour orbital prediction in radial component were better than $1 \mathrm{~m}$, which was evaluated SLR data. User equivalent range error (UERE) was better than $1.5 \mathrm{~m}$, and the precision was stable. The orbit recovery of of GEO of partial subsatellite point was come ture.
\end{abstract}

\section{Introduction}

With the constraints of geostationary orbit, geostationary satellite(GEO) satellite is adopted extensively in satellite navigation area. Especially GEO satellite of partial subsatellite point, it not only can communicate information, but also can boost up the geometric dilution of precision(GDOP), it is an indispensable component in regional navigation system(COMPASS). Because of partial subsatellite point, POD for this kind of GEO become the hotspot and difficulty. he movement scope of GEO relative to a fixed point in earth fixed coordinate system must be among $\pm 0.1^{\circ}$ in longitude and latitude direction, the periodic orbit maneuver must be carried through. The period of orbit maneuver usually is about 30 days. As to this frequent orbit maneuver, the precision and availability of navigation system service will be seriously affected if the precision of satellite can't be improved during orbit maneuver and orbit recovery. The main factors affected the orbit precision are as follows: one hand, ground tracking geometry condition of GEO satellite is nearly unchanged due to regional tracking network, and certain stations can't observe this kind of GEO. The dynamic restriction of stations for GEO satellite is very week. The other hand, the current measurement technique for GEO satellite contains distinct systematic error or ranging biases. So, the time to recover orbit for GEO is about $24 \mathrm{~h}$, the satellite's availability is obviously decreased, service precision of Beidou system(BDS) is affected[1]. 
Three conventional GEO tracking techniques are the pseudoranging, C-band transfer ranging and satellite laser ranging(SLR). The transfer ranging is a two-way ranging mode. The $\mathrm{C}$-band ranging signal from a ground station is transmitted by a transfer onboard GEO satellites designed for communication, and is also received by the station, without clock corrections in the ranging data, its precision is high ${ }^{[2]}$. The precision of SLR is high, and doesn't contain obvious systematic error, but can't get observation in whole day. The L-band downlink navigation signal is received by the receiver and the pseurorange is observed with clock corrections of both the satellite and the receiver, it is easy to come true and spread[3][4][5].

As to the orbit determination based on pseudorange, overseas satellite navigation system currently adopts the uniting estimation method of orbit and clock offset[6]-[13]. But Compass regional satellite navigation system adopt the stations in China, with the constraints of geostationary orbit, orbit parameters and clock parameters can't be effectively separated, the strong correlation of clock parameters and other parametes results in the morbidity of normal equation, we can't effectively estimate the satellite and station combination clock offset. The radio comparison method is widely employed in the satellite navigation system for the satellite-station time synchronization, which is an independent technique. The clock offset is deducted from pseudorange in order to separate the orbit and clock, then only estimate the orbit parameters, which is the best method to determine the orbit for GEO satellite of partial subsatellite point in COMPASS system. GuoRui and SongXiaoyong present POD based on time synchronization mode, its principle is that adopting satellite-earth wireless two-way method and $\mathrm{C}$ wave band satellite two-way method to get high precise satellite clock and station clock, which are deducted from pseurorange, then determine the orbit. In this method, systematic error is the key ingredient to restrict the orbit precision. It need SLR data to demarcate the equipment time delay, so this technique strongly relys on SLR. At the same time, the tracking geometric condition is restricted the distribution and number of time synchronization stations[1] [14]. As a result, an absolute orbit determination method is needed to get rid of the time synchronization stations and SLR.

This paper bringed forward a orbit rapid recovery method based on satellite clock offset and station clock offset. The satellite-ground time synchronization were realized by satellite-earth wireless two-way method, ground-ground time synchronization were realized by constellation combining orbit determination method. The precise orbit determination was realized using pseudorange and phase data without clock offset. A new method of parameters decorrelation based on prior restriction and a short-arc orbit determination method based on restriction by long-arc orbit determination were brought forward.

\section{Orbit Determination Method and Estimated Strategy}

In the dynamical POD, both the quality of dynamical models and the parameter estimation are the two main factors that influence the POD precision. According to the feature, we may divide the force acting on the satellite into three groups: the center gravity from the Earth and the satellite, the conservative acceleration and non-conservative acceleration. The conservative acceleration includes the non-spherical perturbation acceleration, the tidal perturbation acceleration, including the solid earth tide, the ocean tide and the atmospheric tide, the rotational deformation acceleration due to polar motion, the relativistic perturbation acceleration, non-conservative acceleration includes the solar radiation pressure perturbation, the Earth radiation pressure perturbation, the satellite thermal radiator perturbation. JGM-3 Earth gravitation model truncated to 10 by 10 degree and order, JPL DE403 planetary ephemeris, IAU80 nutation model, Box-Wing solar radiation pressure model, and IERS96 solid tide model are employed in the POD[15].

With orbital pre-processing, measurement errors are corrected. Firstly, the antenna phase offset errors of tracking stations are corrected, secondly reductions of satellite antenna phase center to satellite mass center are made, and finally propagation errors are corrected. The tropospheric errors are corrected by using the Saastamoinen model, and the ionospheric errors are corrected by using global precise ionosphere delay model provided by CODE. The satellite-earth wireless two-way method is adopted to get high precise satellite clock and multi-satellite precise orbit determination(MPOD) method is adopted to get high precise station clock, whose precision all are better than $2 \mathrm{~ns}$. As long as anyone station participates in MPOD, we can get high precise station clock, this fundamentally gets rid of time 
synchronization station, the satellite tracking geometric conditions is greatly improved, this is one advantage of the proposed method.

The concrete strategy of POD method based on multi-satellite orbit determination is as follows: pseurorange is smoothed by phase data. The estimated parameters include initial orbit, solar radiation pressure parameter, common systemic error, sine and cosine value of $\mathrm{T}$ orientation empirical force.

The labor of all parameters is as follows: firstly, the common systematic error is designed to absorb the error of inconsistent benchmark between satellite clock and station clock. The benchmark of satellite clock is time of communication antenna's 1pps port, that of station clock is the time of receiver.

Secondly, in single-satellite orbit determination, the solar radiation pressure model is simple ball model, whose precision can't satisfy the demand of orbit precision for regional satellte. The most potential of the solar radiation pressure is as follows:

$$
R=\beta r[A \cos f+B \cos f]
$$

Thereinto, $\beta$ is correlative with reflectance, $A, B$ is correlative with directional parameters of orbit plane. From formula (1), the solar radiation pressure is correlative with orbit period. The empirical force can be adopted to absorb the error of the solar radiation pressure. At the same time, if R,T,N empirical force are synchronously estimated, overfull parameters can result in the strong correlation among parameters and serious morbidity of normal equation. The extent of $\mathrm{T}$ orientation is larger, so only the sine and cosine value of $\mathrm{T}$ orientation empirical force are estimated. The $\mathrm{T}$ orientation empirical force is as follows:

$$
T=a \cos (f+\omega)+b \sin (f+\omega)
$$

$a, b$ is the estimated parameters.

\section{Analysis of Orbit Determination Precision}

\section{Orbit Determination Based on Multi-satellite Orbit Determination}

We make experiments on GEO on $160^{\circ} \mathrm{E}$. The five stations include beijing, a certain station in hainan, a certain station in sichuan, a certain station in northeast, a certain station in southeast. The arc is 24 hours. Table 1 represents the residual error and the orbit accrucy in radial component and the 2-hour orbital prediction errors, which was evaluated with SLR data.

\begin{tabular}{|c|c|c|c|c|c|}
\hline \multirow{2}{*}{ Time } & \multirow{2}{*}{$\begin{array}{l}\text { Stations } \\
\text { num }\end{array}$} & \multirow{2}{*}{ residual error } & \multirow{2}{*}{ num of SLR } & \multicolumn{2}{|c|}{$\begin{array}{l}\text { orbit accrucy evaluated } \\
\text { with SLR data }\end{array}$} \\
\hline & & & & orbit accrucy & $\begin{array}{c}2 \mathrm{~h} \\
\text { prediction }\end{array}$ \\
\hline 2013-03-01 & 5 & 0.618 & 6931 & 0.770 & 1.051 \\
\hline 2013-09-04 & 5 & 0.546 & 878 & 1.547 & 2.162 \\
\hline 2013-09-22 & 5 & 0.865 & 1254 & 2.478 & 2.978 \\
\hline
\end{tabular}

Tab. 1 Residual Error and the Orbit Accrucy in Radial Component(Unit: m)

Table 1 indicates that: the orbit accuracy evaluated with SLR data is better than $1 \mathrm{~m}$ only in 2013.3.1. Those in other two days are larger than $1 \mathrm{~m}$. The orbit precision isn't stable. There are relativity in different extent among $\mathrm{T}$ orientation empirical force, common systematic error, orbit parameters and solar radiation pressure parameters. If we reduce the estimated parameters, depress the relativity, can the orbit precision be stable? We bring forward the decorrelationship method based on priori restriction, through fixupping the best Bias0 based on the UERE, making orbit determination again. If all the orbit precision is lower, we can recalculate the accurate Bias0 through UERE. The calculating method is as follows: accurate $\operatorname{Bias} 0=$ calculative $\operatorname{Bias} 0$ - the value of UERE. If the value of UERE is $3 \mathrm{~m}$, the calculative Bias0 is $3.5 \mathrm{~m}$, the accurate Bias 0 should be $1.5 \mathrm{~m}$. The relativity of parameters is depressed, the orbit precision should be stable. 
The whole orbit determination includes two iterations, namely two-step method in this paper. Firstly, all satellite clock offsets are fixed to their values obtained with two-way frequency and time transfer between satellites and BDT, all station clock offsets are fixed to their values obtained with MPOD, Pseudorange is transferred to range. The estimated parameters include initial orbit, solar radiation pressure parameter, common systemic error. Secondly, UERE of the first step's orbit is obtained, and the mean of UERE is treated as systemic error of the clock offsets. The third step of POD uses the observational data with the systemic error removed in order that the means of UERE of all satellites are close to 0 .

At the same time, we analyze the influence of empirical force in orbit determination, four strategy are constituted:

First strategy: the estimated parameters are initial orbit, solar radiation pressure parameter, common systemic error, sine and cosine value of $\mathrm{T}$ orientation empirical force.

Second strategy: the estimated parameters are initial orbit, solar radiation pressure parameter, common systemic error.

Third strategy: the estimated parameters are initial orbit, solar radiation pressure parameter, sine and cosine value of $\mathrm{T}$ orientation empirical force.

Fourth strategy: the estimated parameters are initial orbit, solar radiation pressure parameter.

Figure 1 represents the orbit accrucy evaluated with SLR data in 2013.9.4. -1 represents 1 hour from the ending time of observation data to the starting time of SLR, 1 represents 1 hour from the ending time of SLR a to the ending time of observation data.

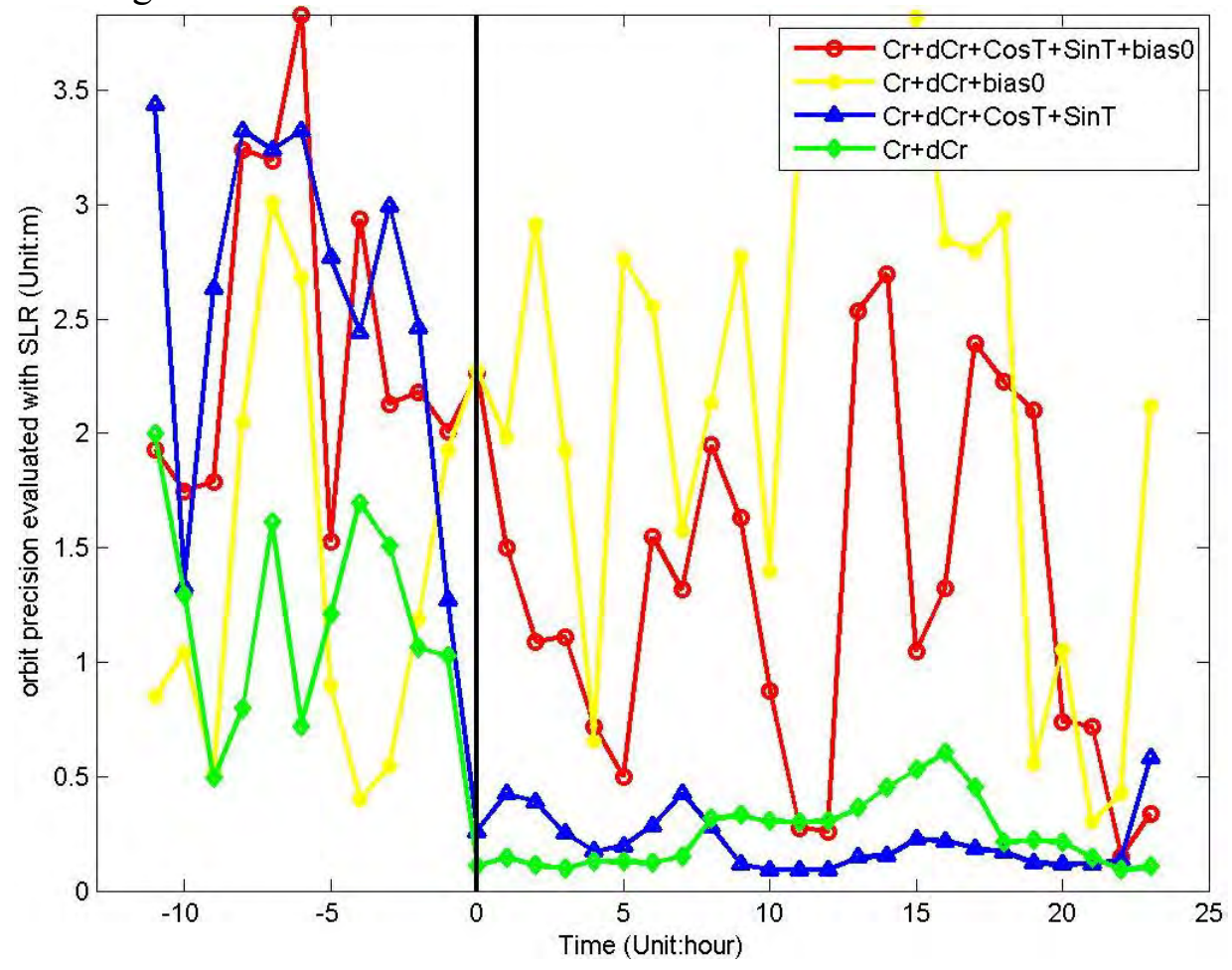

Fig. 1 Orbit Accrucy Evaluated with SLR Data in 2013.9.4

(Black Beeline Represents the Starting Time of SLR)

Fig1 indicates that:

1) the orbit precision based on first strategy isn't stable, orbit precision some times is better than $1 \mathrm{~m}$, some times is larger than $2 \mathrm{~m}$, the 2-hour orbital prediction error is about $2 \mathrm{~m}$.

2 ) the orbit precision based on second strategy is high and stable, but the orbital prediction error is larger with the time, so when we estimate the empirical force parameters in orbit determination, the orbital prediction will be low.

3) Bias0 coming from the results of first strategy is adopted in third strategy and fourth strategy. The orbit precision based on fourth strategy is highest and stablest. The orbit precision and the 2-hour orbital prediction all are better than $1 \mathrm{~m}$. 


\section{Short-arc Orbit Determination Method Based on Restriction by Long-arc Orbit Determination}

As to the orbit recovery after maneuver, the estimated parameters include initial orbit, solar radiation pressure parameter, the solar radiation pressure parameters are constrainted by the weighted estimation method.

UERE is an important index parameter for evaluating the service capability of a navigation system, summarizing the total errors of the space segment (satellite orbits and clock offsets), the propagation segment (ionospheric and tropospheric delay) and the user segment (multipath and random errors). When the precision of every error source except the orbits is acceptable, UERE can be adopted to evaluate the precision of the orbits. For a receiver with known coordinates and clock synchronous with BDT, UERE is as follows:

$$
\begin{aligned}
\text { UERE }= & \rho-\left(R\left(t_{k}-\delta t_{r}-\tau, t_{k}-\delta t_{r}\right)+C \delta t_{r}-C \delta t_{s}+\Delta D_{\text {trop }}\right. \\
& \left.+\Delta D_{\text {iono }}+\Delta D_{\text {rel }}+\Delta D_{\text {ant }}^{r}+\Delta D_{\text {ant }}^{s}+\Delta D_{\text {tide }}\right)
\end{aligned}
$$

where $\rho$ is the pseudo-range measurement, $R$ is the geometric range between satellite and receiver, $\delta t_{r}$ is the receiver clock offset (which is 0 in this case), $\delta t_{s}$ is the satellite clock offset in the navigation message, $\tau_{r}, \tau_{s}$ are respectively the equipment time delay of the receiver and satellite, $\Delta D_{\text {trop }}, \Delta D_{\text {iono }}$, $\Delta D_{\text {rel }}, \Delta D_{\text {ant }}^{r}, \Delta D_{\text {ant }}^{s}, \Delta D_{\text {tide }}$ are respectively tropospheric delay, ionospheric delay, relativistic effects delay, antenna phase center delay of satellite and receiver, and tidal effects delay.

Table 2 gives the precison of UERE for $160^{\circ}$ E GEO based on 4 hours data after orbit maneuver.

Tab. 2 Precison of UERE for GEO 4 Hours after Orbit Maneuver

\begin{tabular}{ccc}
\hline Beginning time of orbit maneuver & End time of orbit maneuver & UERE(RMS) \\
\hline 2013-03-01 19:00 & $2013-03-0121: 00$ & 1.222 \\
2013-09-04 04:00 & $2013-09-0406: 00$ & 1.061 \\
2013-09-22 07:00 & $2013-09-22$ 08:00 & 0.557 \\
\hline
\end{tabular}

Table 2 shows that UERE is better than $1.5 \mathrm{~m}$, and the precision is stable.

\section{Conclusions}

This paper analyzed the orbit determination of GEO satellite of partial subsatellite point. The orbit determination method based on multi-satellite orbit determination is systematically analyzed. The advantage of this method is as follows: firstly, this method doesn't rely on the number and distribution of stations, secondly, we can estimate systematic error and other parameters in orbit determination, then get the exactest value of systematic error based on UERE evaluation, which make this method get rid of the restriction of SLR. The main conclusions are as follows:

1) The decorrelationship method based on priori restriction can make the orbit prediction stable, the experiment results show that the orbit precision and the 2-hour orbital prediction all are better than $1 \mathrm{~m}$.

2) A new method of a short-arc orbit determination method based on restriction by long-arc orbit determination is brought forward. The solar radiation pressure parameters are constrainted by the weighted estimation method, systematic error was fixed. Priori values of the kinematics and dynamics parameters are derived from the long-arc orbit determination. The time of orbit recovery after maneuver is shortened from $24 \mathrm{~h}$ to $4 \mathrm{~h}$. The 2 -hour orbital prediction in radial component are better than $1 \mathrm{~m}$, which is evaluated SLR data. User equivalent range error (UERE) is better than $1.5 \mathrm{~m}$, and the precision is stable. This method has been successfully applied in the engineering construction of BDS, the system availability and service performance are greatly improves.

\section{Acknowledgement}

This research was financially supported the National Natural Science Foundation of China (Grant Nos. 
41204022), the Opening Project of Shanghai Key Laboratory of Space Navigation and Position Techniques (Grant No. 12DZ2273300 and Grant No. 13DZ2273300) and Surveying and Mapping Basic Research Program of National Administration of Surveying, Mapping and Geoinformation (Grant No. 13-01-06).

\section{References}

[1]Guo rui, Liu li, Li xiaojie. Precise Orbit Determination for GEO Satellites Based on Both Satellite Clock Offsets and Station Clock Offsets [J]. chinese Journal of space science, 2012, 32(3): 406-411.

[2]Huang yong. The Orbit Determination in Orbit Maneuver Based On CAPS Data[J]. SCIENCE CHINA Physics ,2008,38(12): 1750-1758.

[3]GUO Rui, HU XiaoGong, et al. Precise Orbit Determination For Geostationary Satellites With Multiple Tracking Techniques[J]. Chinese Sci Bull, 2010, 55: 428-434.

[4]Li Z G, Yang X H, Ai G X, et al. A New Method For Determination Of Satellite Orbits By Transfer Data[J]. Sci China Ser G: Phys Mech Astron, 2008, 38(12): 1711-1722.

[5]ZHOU ShanShi, HU XiaoGong, WU Bin, et al. Orbit Determination And Time Synchronization For a GEO/IGSO Satellite Navigation Constellation With Regional Tracking Network[J]. SCIENCE CHINA Physics, Mechanics \& Astronomy, 2011, 54(6): 1089-1097.

[6]E. D. Kaplan,C. J. Hegarty, GPS Principle And Application(second edition)[M], electron industry publishing company, 2008.

[7]Global Positioning System Precise Positioning Service Performance Standard,Department of Defense. 2007.

[8]Global Positioning System Standard Positioning Service Performance Standard. Department of Defense. 2008.

[9]GPS Operational Performance Report For Calendar Year 2008, GPS Operations Center, 2009.

[10]Xu guochang, GPS Theory, Algorithms, and Application (2nd Edition) [M], Springer, 2007, 50-80

[11]Xu guochang, Orbits[M], Springer, 2007, 35-79.

[12]O. Montenbruck, E. Gill. Satellites Orbits - Models, Methods, and Applications [M]. Germany: Springer,2000.05:312-318.

[13]Ollie Luba, Larry Boyd, Art Gower, GPS III System Operations Concepts[J], IEEE A\&E SYSTEMS MAGAZINE. JANUARY 2005:290-305.

[14]Song xiaoyong, jia xiaolin, mao yue. Single-satellite Orbit Determination Based On Time Synchronization Stations [J]. surveying and mapping science, 2009, 34(5): 8-10(In Chinese).

[15]Guo rui, $\mathrm{Hu}$ xiao gong, Liu Li. Orbit Determination for Geostationary Satellites with the Combination of Transfer Ranging and Pseudorange Data[J]. SCIENCE CHINA Physics, Mechanics \& Astronomy, 2010, 40(8): 1054-1062. 